\title{
The Rhetoric and Translation of English Advertisement
}

\author{
Xiang Xu \\ College of Foreign Language, Qingdao University of Science and Technology, Qingdao 266061, China \\ E-mail:stephenxu10@163.com
}

\begin{abstract}
Advertisement is the major way of promoting sales. To achieve this aim, the AD language gets used to reinforce the effect with the help of rhetoric devices such as simile, metaphor, personification, pun, rhyme, etc. In term of the difference between English and Chinese, some translation methods including Literal Translantion, Free Translation and Modulation could be skillfully used during the time of translating rhetoric devices. Because almost all kinds of subject knowledge and commerce knowledge are involved in translation course, not only should the translator have abundant language skills and fertile fanciful abilities, but also understand commerce psychology and sales strategy. Thus, the translation contents could be pricisely restored. With the beautiful words and expressions and the easiness of reciting aloud, the final version of translation can be able to serve the purpose of promotinmg sales.
\end{abstract}

Keywords: English advertisement, Rhetoric, Translation

\section{The significance of studying English Advertisement}

The forming of the word Advertisement dates back to Lantin language Adverture, with its meaning of "attaction". In the middle ages of English (around 1302-1475), it varied into the word Advertise, and the meaning also changed into "attracting somebody to notice something", or "informing somebody so as to attract him". Until the end of the $17^{\text {th }}$ century, England set out to hold commercial activities in great dimension. At that time, the word Advertisement became popular and used widely. The meaning of advertisement refers to not only one piece of advertisement, but a series of advertising activities. Advertisement Time Weekly defines the word, "individual, commodity, labour, campaign, regarding printing, handwriting, dictating or painting as presenting way, paied by advertiser to make public propaganda to promote its aim by sale, usage, vote, or agreement." In modern societies, following the deepening and developing of the international work dividing and the daily strengthening of the tendency according to the one system of global economy, the communication of commodities among the world gets more frequent. In order to catch hold of the markets, each country competes to promote his products. One of the best methods is to introduce national goods and producer's reputation by advertisement. So the study of the advertisement through many view angles is sure to be the focal point of linguistics and translation.

The advertisement language is named as commercial language, belonging to the area of advertisement in written form. This form consisits of five parts, including headline, bodytext, slogan, trademark and illustration. The former three being verbal and the latter two non-verbals. This paper aims at the study of the rhetoric devices about verbal part and the translating problems they cause.

\section{The significance of the rhetoric devices in advertisement and the skill of translation}

Rhetoric is a science of studying language art. It chooses appropriate language method and expressing way chiefly according to communicating contents and language settings, etc. As a science whose purpose is the research for the regulation rule of the effects of language expression, it cannot be easily divided by the art of advertisement language. The key to study the effects of Rhetorics in advertisement is to be familiar with the rhetoric devices and their usage. The rhetoric device is the name of the rhetoric skills in language types, being the core of Rhetoric. It concludes simile, metaphor, personification, pun, hyperbole, antithesis, etc, usually being used for the stress of atmosphere and colour.

Language is one part of language and a carrier of culture. It reflects a nation's characteristics, ont only containing the background of this nation's history and culture, but their life view, life style and thinking way. In the constant development of society and culture, the two nations, England and China, have formed their special own rhetoric devices. There are many same or different places when they are compared with each other. The difficulty in the rhetoric devices of English and Chinese translation comes into being, owning to their difference among many sides, such as word forming, word style changing, syntax structure, language order, and so on, also adding the difference from word matching area and language rhythm.

From the view of translation, the rhetoric devices may be divided into three types: able, hardly able, disable (to be translated). According to this condition, "we'd better find proper way that can express the original meaning to conduct 
its best effect perfectly into the translated script.”(Feng Qinghua, 2002:151) according to Teng Weiguo, the three principles of translation are honesty, simpleness and vivdness.

The three methods of translation of the rhetoric devices are Literal Translation, Free Translation and Modulation.

\subsection{Literal Translation}

Literal Translation refers to the perfect keeping for the contents and styles of the original, especially its simile or metaphor, image and national elements. The leading condition is not to disobey the laguage rules and cannot be easily caused by false imagination. Literal Translation can be used safely when there is some common nature in Chinese and English, especially there is some corresponding device.

\subsection{Free Translation}

Free Translation indicates the method for mending the blank of word lacking and the difference of language structure. Thre are quite a few devices interfering with unique language feature and habits, or culture custom, according to different background which cannot be familiar with language importing countries. Thus it drives the translator to search for their own national idioms, selecting proper sentences or phrases to send the message or main meaning which are faithful to the original scripts.

\subsection{Modulation}

Modulation should be mastered from grand view. It's hard for us to express the devices completely in a translated script, so the traslator is supposed to deal with them from the whole text, adding or deleting some places, particularly using rescuing method to cope with some disable translated parts.

This paper stresses on the types of the rhetoric devices in English Advertisement and how to translate them.

\section{The rhetoric in English Advertisement}

Advertisement is called sub-literature. In order to conduct effective message of commodity, the advertisement language is pursuing the aim at attracting more customers by colorful and various advertisement language.

\subsection{Simile}

Simile shows the similarity of the two different things - the describing object and the described object. In English, it is connected with Like and as, in Chinese corresponded by "hao xiang"or "wan ru". The first example is an advertisement about spectacles: Featherwater, light as a feather.Feather is its brand,and "light as a feather"can be Literal Translated as "qing ru hong mao". This advertisement touches the reader by the spectacle's feature-light weight, which is used so vividly. Another baverage advertisement is "Breakfast without orange juice is like a day without sunshine". Only being literally translated into "Mei you ju zhi de zao can jiu xiang meiyou yang guang de ri zi"can the function of the products be presented vividly and charmingly to the face of the reader with the characteristics of touching his heart.

\subsection{Metaphor}

Metaphor indicates the superposition of the describing object and the described object. In the sentence the two things with commom feature or inner connection, but only one appears in the text. They usually be connected with the verb "to be". Given four examples of commercial advertisement, no exception of using "life is."

Nissan car: life is a journey. Enjoy the ride. (Sheng huo jiu shi yi ci lu xing, zhu nin lu tu yu kuai);

Gatorade drink: life is a sport, drink it up. (Sheng huo jiu shi yi chang yun dong, he xia ta);

Tequila liquor: life is harsh, your tequila shouldn't be.(sheng huo shi ku se de, er nin de tequila jiu que bu shi;

Wolderness system: life is discovery, and we have directions to get you there.(sheng huo jiu shi fa xian, rang wo men qu fa xian ba )

The above four sentences connect the describing object with life; using the link verb "is"directly. The pattern of the translation is almost the same as the original, simple but easy to catch the reader's imagination.

Here is a liquor advertisement: Sophisticated, sweet-to-drink Pink Lady. (gao ji ke kou de hong fen jia ren ). The simple words make us feel that a pretty girl wearing pink is coming to us flirtatiously. Another lipstick advertisement is: The most sensational place to wear satin on your lips. The script describes the lipstick as satin. The colour on one's lips is just as bright and soft as satin. Someone translates it into "si ban rou hua de kou hong ca zai chun shang zhe ge zui fu you ji qing de di fang". it seems nothing left untranslated, however, the rhyme is a little less than the original. The styles of modern poem can be used to translate it as "si ban rou hua de kou hong, ca zai ning ju ji qing de chun shang". The superlative degree "most" cannot be corresponded by "zui", but the word "ji" expresses the meaning precisely, and another kind of rhyme appears.

\subsection{Personification}

Personification is the device to describe some non-humans as real human beings. They've given human's emotion or 
action to achieve the requirement of being vivid. This skill is usually carried out so as to make the commodity alive, full of emotion, easy to be near by people. For example, "She has her own spirit and it graces everyone she comes near". This advertisement personifies the perfume. The word "she"refers to not only the representative beauty, but the perfume itself, and women are the only users. This sentence can be richer than directly shows the advantanges of products. The quality of this advertisement can touch those women who are pursuing beauty desperately.

\subsection{Pun}

Pun shows that one word suggests two or more meaning or different imagination. It seems like a word game, being the traditional rhetoric feature of English advertisement. For example, Give your hair a touch of spring.(gei ni de tou fa yi lu shun se). Here, the word spring has double meanings. At one side means the spring of hair, at another, prosperous sping season. As for the translation, "zhan" is corresponded with touch, and "chun" is followed by "se". another example, From sharp minds.Come Sharp products.(xia pu chan pin lai zi min jie de si wei).it is well-known that "sharp" here means both the copy machine and the meaning of being smart and not blunt.

\subsection{Rhetocical Qustion}

Rhetocical Qustion means that the author set out a question on purpose though he knows the answer well. Three conditions are included: ask and answer by oneself, ask but not answer by oneself and complete retocical question.

Here is a chocolate advertisement,

Have you ever noticed what a remarkable effect Godiva Chocolate has on people?

The mere glimpse of the scintillating gold box foretells of many luscious moments to come--

(ni ke ceng xiang dao Godiva qiao ke li dui ren de ying xiang you duo da ma? Zhi yao pie yi yan na jin guang shan shan de he zi, li ke jiu hui gan dao xiang wei you ren----)

The answer is properly correlated with the question, vividly and full of curiosity to the reader.

Another black hair spray: Are you going gray too early? (nin de wu fa shi fou bai de you dian tai zao?) The rhetoric questions are perfectly placed in above classic examples.

\subsection{Rhyme}

Rhyme is one of the skills of English poem, roughly divided into two types-alliterration and end rhyme, making proper rhythm for the reader to recite.

\subsubsection{Alliterration}

Alliterration refers to the repetition of similar sounds, usually consonants or consonant clusters in a group of words. Usually this occurs at the beginning of those words. Alliterration in advertisement looks eyes-catching, and sounds pleasant to the ears. There are two examples,

Spare,shapely and sensational—one step dressing.(jie sheng, yun chen, ji qing—yi bu qun);

Health, humor and happiness - It's a gift we'd love to give. (jian kang, you mo, kuai le --- zhe jiu shi wo men gei nin de li wu).

The repetitions of allteration in the two sentences appear with the value of notice and memory, easily producing strong emotional effects. The space of readers imagination is produced.

\subsubsection{End Rhyme}

If both rhyming words occur at the ends of lines, it's called end rhyme. It also makes a series of sentences sound vivid. Here is the advertisement of lady's swimming suit --Flash, dash, Classic splash. (shan guang, xuan yao, jing dian de fei jian). Three vowel [s]s construct a wonderland of lotus diving into water colourfully and spalshed drops flying everywhere. Another product's promoting advertisement: Big thrills, small bills (da ci ji, xiao hua fei). The two consonant[s]s also builds some special taste. So, end rhyme adheres some features of being easily recognized and artistically superposed. Then the atmosphere is constructed successfully.

Besides the types mentioned above, other rhetoric devices in English advertisement are hyperbole, parallelism, repetition, antithesis, and so on. Those are supposed not to be discussed owing to the length limitation.

\section{Conclusion}

This paper is mainly about the rhetoric devices and translation of English advertisement. In the practical process of translation, the best result needs deep considering action, good methods and ability of precise choices. This resaerch can give us more opportunity to understand the feature of English and master the highlight of English advertisement. Having the knowledge of the culture, value opinion and custom, we are certain to advocate national products so as to lauch them in international markets effectively. 


\section{References}

Jin, Qiuhua. (2003). The Characteristics of Advertisement Language, Journal of Liao ning College.

Feng, Qinghua. (2008). A Practical Course for Tanslation, Shanghai: Shanghai Foreign Language Eduction Press

Beckwith, Harry. (1997). Selling the Invisible: A Field Guide to Modern Marketing, Warner Books

Bly, Bob. (1998). Business to Business Direct Marketing: Proven Direct Response Methods to Generate More Leads and Sales, NTC Business Books

Caples, John. (1983).How to Make Your Advertising Make Money, Prentice Hall 